\title{
Complications of Central Venous Access Devices: A Systematic Review
}

Amanda J Ullman ${ }^{1,2,3}$, RN MAppSci, Nicole Marsh',3,4, RN MAdvPrac, Gabor Mihala, ${ }^{3,5,6}$ MEng GCert(Biostat), Marie Cooke ${ }^{1,2,3}, \mathrm{RN}$ PhD, Claire M Rickard ${ }^{1,2,3}$, RN PhD

Affiliations: ${ }^{1}$ School of Nursing and Midwifery, Griffith University, Queensland, Australia; ${ }^{2}$ NHMRC Centre of Research Excellence in Nursing, Centre for Health Practice Innovation, Menzies Health Institute Queensland, Australia; ${ }^{3}$ Alliance for Vascular Access Teaching and Research Group, Menzies Health Institute Queensland, Australia; ${ }^{4}$ Centre for Clinical Nursing, Royal Brisbane and Women's Hospital, Queensland, Australia; ${ }^{5}$ School of Medicine, Griffith University, Queensland, Australia; ${ }^{6}$ Centre for Applied Health Economics, Menzies Health Institute Queensland, Australia

Address correspondence to: Amanda Ullman, School of Nursing and Midwifery, Griffith University, N48 Kessels Road, Nathan, Queensland, 4111, Australia. Tel: +61 (0)7 3735 7854; Email: a.ullman@griffith.edu.au

Short Title: Complications of Central Venous Access Devices

Financial Disclosure Statement: The study authors have no financial relationships relevant to this article to disclose.

Funding source: This research has been undertaken as part of Ms Ullman's $\mathrm{PhD}$ program. She has received PhD scholarship funding from the Menzies Health Institute Queensland, NHMRC Centre of Research Excellence in Nursing and Centurion Medical Products.

Conflict of Interest Statement: Ms Ullman, Ms Marsh and Dr Rickard have received funding from CVAD dressing manufacturers (3M; Carefusion; Centurion Medical Products) but these medical products were not included within the scope of this review.

Abbreviations: BSI=bloodstream infection; $\mathrm{CENTRAL}=$ central register of controlled trials); $\mathrm{CVAD}=$ central venous access device; $\mathrm{CINAHL}=$ cumulative index to nursing and allied health; $\mathrm{CI}$ = confidence interval; $\mathrm{HD}=$ hemodialysis; $\mathrm{IR}=$ incidence rate; $\mathrm{MeSH}=$ Medical subject headings; MOOSE= meta-analysis of observational studies in epidemiology; PICC=peripherally inserted central catheter; PRISMA= preferred reporting items for systematic reviews and meta-analyses; STROBE= strengthening the reporting of observational studies in epidemiology. 


\section{Contributors' Statement:}

Amanda J Ullman: Ms Ullman conceptualized and designed the study, carried out the initial analysis, drafted the initial manuscript, and approved the final manuscript as submitted.

Nicole Marsh: Ms Marsh assisted with the acquisition of data, critically reviewed the manuscript and approved the final manuscript as submitted.

Gabor Mihala: Mr Mihala carried out the subsequent analysis, assisted with the interpretation of the data, reviewed and revised the manuscript and approved the final manuscript as submitted.

Marie Cooke, Claire M Rickard: Drs Cooke and Rickard assisted with the conception and design of the study, interpretation of the data, reviewed and revised the manuscript, and approved the final manuscript as submitted.

All authors approved the final manuscript as submitted and agree to be accountable for all aspects of the work. 


\begin{abstract}
:
Context: The failure and complications of central venous access device (CVAD) result in interrupted medical treatment, morbidity and mortality for the patient and the risks and resources associated with the insertion of new CVAD.
\end{abstract}

Objective: To systematically review existing evidence of the incidence of CVAD failure and complications across CVAD types within pediatrics.

Data Sources: CENTRAL, PubMed and CINAHL databases were systematically searched to January 2015.

Study Selection: Included studies were of cohort design and examined the incidence of CVAD failure and complications across CVAD type in pediatrics within the last ten years. CVAD failure was defined as CVAD loss of function prior to the completion of necessary treatment, and complications as CVAD-associated bloodstream infection, CVAD local infection, dislodgement, occlusion, thrombosis and breakage.

Data Extraction: Data were independently extracted and critiqued for quality by two authors.

Results: Seventy-four cohort studies met the inclusion criteria, with mixed quality reporting and method. Overall, 25\% of CVADs failed prior to completion of therapy (95\% CI 20.9\%-29.2\%) at a rate of 1.97 per 1,000 catheter days (95\% CI 1.71-2.23). The failure per CVAD device was highest proportionally in hemodialysis catheters (46.4\%, 95\%CI 29.6\%-63.6\%); and per 1,000 catheter days in umbilical catheters (28.6 per 1,000 catheter days, 95\% CI 17.4-39.8). Totally implanted devices had the lowest rate of failure per 1,000 catheter days (0.15, 95\% CI 0.09-0.20).

Limitations: The inclusion of non-randomized and non-comparator studies may have affected the robustness of the research.

Conclusions: CVAD failure and complications in pediatrics is a significant burden on the healthcare system internationally. 


\section{Background}

Worldwide, millions of central venous access devices (CVADs) are used in healthcare facilities to provide supportive and interventional therapies during acute and chronic illness. Within pediatrics, the therapies that CVADs facilitate are diverse, varying from the life-long administration of nutrition, to the aggressive treatment of oncological conditions.[1] Children with CVADs are already vulnerable to complications and disability because of their underlying health condition. This vulnerability to complications is worsened by the risk of adverse events associated with the insertion and management of CVADs. [2, 3]

There are a range of CVADs available, which healthcare professionals elect to insert on the basis of the predicted duration of clinical necessity (short, medium or long term), risk of adverse outcomes, treatment requirements (e.g. hemodialysis), frequency of usage and vein availability. Traditionally, non-tunneled and umbilical CVADs have been recommended for short term usage (up to 7-10 days), [4-6] peripherally inserted central catheters (PICCs) for short to medium term usage (4 weeks to 6 months), [4, 6] and tunneled CVADs and totally implantable catheters for long term usage (months / years). [6, 7] The goal for all CVADs is to provide safe and reliable vascular access to facilitate necessary treatment, without complications related to insertion, maintenance and/or removal.

\section{CVAD failure}

CVADs provide a vital contribution to each child's treatment, and their failure can result in significant harm. Each failure also places a significant burden on the healthcare system. The immediate interruption to necessary treatment results in an inability to receive prescribed chemotherapy, fluids, nutrition, antibiotics or other necessary medicines. [8, 9] CVAD reinsertions are costly, requiring highly skilled staff, large amounts of sterile and disposable equipment, theatre time, monitoring devices and radiological confirmation of placement.[10] Their insertion can result in complications including pneumothorax, arterial puncture, hemorrhage and cardiac rhythm Page | 4 
dysfunction [11], with overall CVAD insertion-related complications reported in $7 \%$ to $18 \%$ of CVAD insertions. [12, 13] The more CVADs a child has previously had inserted, the more complex the procedure becomes, as CVAD failures can result in venous damage and insufficiency. [12] Even after successful CVAD insertion, there are many mechanisms which may result in CVAD failure or complication, many of which are considered preventable. [14, 15]

\section{Infectious complications}

CVADs place patients at risk of local and systemic infectious complications, including local site infection (e.g. exit site) and bloodstream infection (BSI). [5, 16] The multifocal path of microbial transmission of bacteria or fungi can be as a result of skin organisms at the insertion site, contamination of the internal device hub, hematogenous seeding and/or infusate contamination. [5, $17,18]$ Microbial colonization of the entry or exit site of CVADs can result in local infection. This infection is commonly caused by resident skin flora, and results in inflammation of the skin (dermatitis), subcutaneous tissue (cellulitis) or vein (phlebitis). CVAD failure related to local infection is normally due to poor response to topical therapy, tunnel infection and purulent drainage. [19]

CVAD-associated BSIs are prevalent world-wide, with an estimated 41,000 occurring in United States hospitals each year. [20] CVAD-associated BSI is associated with a prolonged hospital stay ( 10 days) and an increase in the relative risk of death by 1.06 (absolute $1 \%$ attributable increase)

.[21] CVAD-associated BSI have an attributable cost of between \$5,821 and \$60,536USD per event [22-24] and frequently result in device failure.

\section{Mechanical complications}

As CVADs remain partially exogenous to the body, CVAD failure may also occur as a result of dislodgement and breakage. Breakage of a CVAD is most commonly due to the use of excessive force, causing a split in the structure of the device. This may be due to drag from multiple heavy 
infusion tubes, catching on environmental structures (e.g. clothing, bedrails), intentional or accidental removal by patients or the use of inappropriately small syringe size for the injection of infusates.[3]

CVAD occlusion may also result in device failure and is caused by the presence of a fibrin sheath, medication precipitate, catheter tip thrombus or position of the catheter tip against a vessel or chamber wall. $[8,25]$ CVAD-associated thrombosis may be as a result of fibrin deposited inside the CVADs (intraluminal thrombosis), adhering to the vein wall (mural thrombosis) or around the intravascular portion of the CVADs (fibrin sheath). [9, 25] Fibrin sheaths only cause malfunction when the sheath extends around or over the tip of the CVADs, and in many cases CVAD-associated thromboses are asymptomatic and the device continues to function. [25]

Individual studies have examined the rate and incidence rate of CVAD failure and complications in pediatrics, but an overall estimation per CVAD type throughout this population has not been established. This systematic review aims to examine the proportion and rate of CVAD failure and complications in pediatrics across CVADs type.

\section{Methods}

The study used standard methods for systematic review and is reported in accordance with MOOSE (Meta-analysis of observational studies in epidemiology) [26] where applicable.

\section{Eligibility criteria}

A systematic search for cohort studies examining failure and complications of CVADs in pediatrics was conducted. Studies were eligible for inclusion if they met predefined inclusion criteria: (1) cohort design (prospective or retrospective); (2) study participants aged 0-18 years; (3) reported the failure and / or complications of CVADs included as outcome measures (4) reported the outcomes per PICCs, umbilical catheters, non-tunneled percutaneous CVADs, hemodialysis (HD) catheters, Page $\mid 6$ 
tunneled CVADs or totally implantable CVADs. The review was limited to observational studies in order to describe the failure and complications statistics across CVADs in pediatrics, without confounding the description with the comparative effectiveness of various interventions. There were no restrictions placed in terms of patient underlying condition. We excluded studies if they were not written in English and were greater than ten years old, to reflect and maximize relevance to current practices.

\section{Outcome measures}

The primary outcome of the review was defined a priori, in accordance with landmark intravascular research, as CVAD failure prior to the completion of necessary treatment.[27-29] The secondary outcomes were CVAD complications after successful CVAD insertion. These were:

1. CVAD-associated BSI: Minimum definition of a laboratory confirmed bloodstream infection that is not secondary to an infection at another body site, with a CVAD in place for greater than two days; [20]

2. CVAD-associated thrombosis: Development of thrombosed vessel (partial or complete) at the CVADs site diagnosed via ultrasound; [30]

3. Occlusion or blockage: As defined by study investigators and included partial and full blockage of the CVAD lumen or lumens, irrespective of occlusion treatment; [30]

4. Dislodgement or migration: As defined by study investigators and included partial, complete and accidental removal which results in the CVAD tip no longer being placed in the inferior or superior vena cava;[5]

5. Breakage or rupture: As defined by study investigators and included a visible split in CVADs material diagnosed by leakage or radiographic evidence of extravasation from a portion of the CVAD into tissue; [13] and

6. Local infection and phlebitis: As defined by the study investigator and included exit, entrance, tunnel infections and phlebitis.[5] 


\section{Search strategy and study selection}

The Cochrane Central Register of Controlled Trials (CENTRAL; the Cochrane Library), US National Library of Medicine National Institutes of Health (PubMed), and Cumulative Index to Nursing and Allied Health (CINAHL) databases were systematically and independently searched on the $27^{\text {th }}$ of January, 2015. Medical subject headings (MeSH) were developed by a healthcare librarian and were "vascular access devices”, “central venous catheters” and "pediatrics”. Additional studies were identified through searches of bibliographies.

\section{Data extraction and missing data}

All data were extracted by two independent investigators (AU, NM), using a standardized data extraction form. Study data were extracted regarding the number of patients, catheters, patient population, CVADs type, study method, frequency of CVAD failures and complications, catheter days and country of origin. For studies with missing data (e.g. CVAD catheter days), the study authors were contacted via email where possible.

\section{Statistical methods}

As only cohort studies were included, descriptive statistics have been used to provide summative information of the study population and results. Score confidence intervals (CI) with FreemanTukey double arcsine transformations were calculated for individual studies where the outcome was dichotomous (failure/no failure; binomial data),[31] and Poisson confidence intervals and standard errors were calculated for incidence rate (IR) outcomes. Pooled estimates were generated with random-effects meta-analysis, with results summarized per device type using proportion (\%) and 95\% CI. IR outcomes (continuous data) were pooled using inverse variance, with the DerSimonian and Laird method, per 1,000 catheter days and 95\% CI. Heterogeneity (between studies) was assessed using the $\mathrm{I}^{2}$ measure, categorized as low (<25\%), moderate (25-75\%) or high $(>75 \%)$. 
Subgroup analysis was completed with random-effects meta-regression. Subgroup analysis and tests for overall effect (null hypothesis: no treatment effect) were assessed with the P-value, categorized as significant at $<0.05$ level. Extreme or obviously incorrect data were re-checked for accuracy. Stata [32] was used for all analyses.

\section{Subgroup analysis}

Given the predicted heterogeneity of the study populations, subgroup analyses were planned to compare CVAD failure rates by CVADs types in populations involving: neonates and pediatrics; oncology / hematology and all others; and outpatient and inpatient managed devices. Results of the subgroup analyses are described using CVAD failure proportion, IR per 1,000 catheter days and 95\% CI, where possible.

\section{Risk of bias assessment and sensitivity analyses}

In accordance with the MOOSE guidelines,[26] study quality was assessed by examination of key components of an included study's design, in comparison to an overall score. Based upon elements of the STROBE (STrengthening the Reporting of OBservational studies in Epidemiology) guidelines,[33] these design features were clarity, consistency and rigor of the outcome measures, completeness of outcome reporting and research methods. To further describe the risk of bias within the meta-analysis, sensitivity analyses were undertaken comparing CVAD failure proportion and IR per 1,000 catheter days, and 95\% CI per CVADs type between retrospective and prospective studies.

\section{Results}

\section{Systematic search results}


Figure 1 describes the flow of inclusion and exclusion for the study selection, in accordance with the referred Reporting Items for Systematic Reviews and Meta-Analyses (PRISMA) guidelines. [34] After removal of duplicates, 307 records were identified with 96 requiring full text for review. From the full text articles, 22 were excluded as they included both adult and pediatric participants, [35, 36] reported intraoperative CVADs complications, [11, 37-39] did not provide separate outcome data per CVADs type, [7, 40-44] provided inadequate information to facilitate data extraction, [45-51] or had outcome definitions not in accordance with the review. [52-54] Seventyfour studies were assessed as meeting the inclusion criteria.

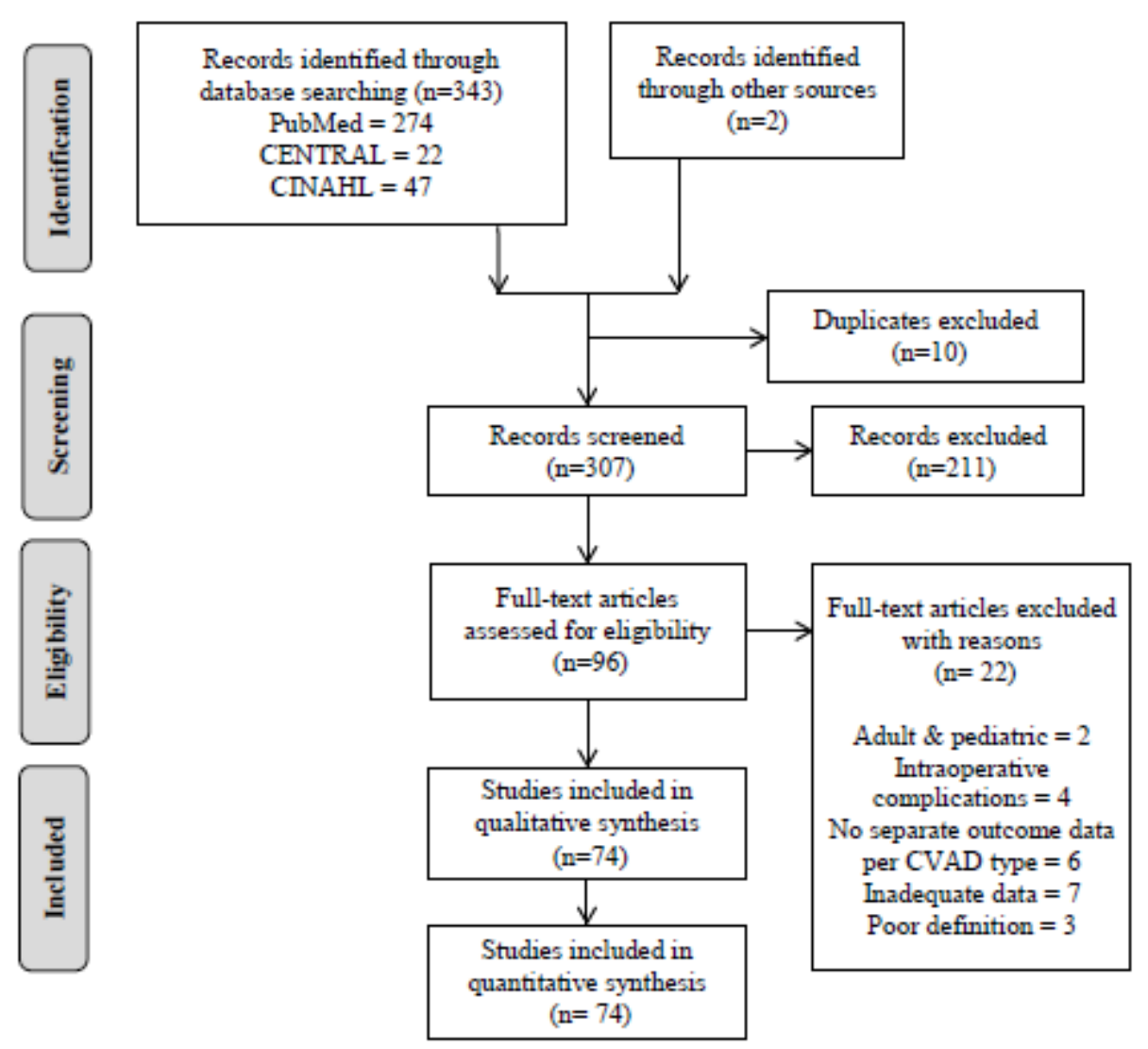

Figure 1: PRISMA flow chart of study selection

Thirty-two study authors were contacted to provide additional information regarding the research results; most commonly for the total CVAD catheter days per CVAD type. Eleven authors were able to provide the additional information,[55-65] three were unable to provide the requested data, [66-68] and 17 did not respond.[69-85] 
The review includes 24 prospective and 50 retrospective cohort studies. These studies were undertaken in Europe, [2, 3, 30, 57-60, 66, 68, 71, 73, 75, 77, 79-81, 86-99] North America, [62-65, 67, 72, 76, 83-85, 100-114] Asia, [55, 61, 69, 70, 74, 82, 115-125] and South America. [56, 78] Included subjects were aged 0-17 years and required treatment for oncological or hematological conditions, support during intensive care admission, or central access for hemodialysis, postsurgical, general infusion therapy and parenteral nutrition. Table 1 describes the populations and CVADs types described within the included studies.

Table 1: Number of included studies with patient population and CVAD type

\begin{tabular}{|c|c|c|c|c|c|c|}
\hline CVAD Type & $\begin{array}{c}\text { Neonatal } \\
\text { Intensive } \\
\text { Care } \\
\end{array}$ & $\begin{array}{c}\text { Pediatric } \\
\text { Intensive } \\
\text { Care } \\
\end{array}$ & $\begin{array}{c}\text { Hematology / } \\
\text { Oncology }\end{array}$ & $\begin{array}{c}\text { General } \\
\text { Pediatrics }\end{array}$ & $\begin{array}{l}\text { Outpatients } \\
\text { (including } \\
\text { GE failure) }\end{array}$ & TOTAL \\
\hline $\begin{array}{l}\text { Peripherally } \\
\text { inserted central } \\
\text { catheter } \\
\text { (PICC) }\end{array}$ & 16 & - & 6 & 8 & 3 & 33 \\
\hline $\begin{array}{l}\text { Umbilical } \\
\text { catheters }\end{array}$ & 5 & - & - & 1 & - & 6 \\
\hline $\begin{array}{l}\text { Non-tunneled, } \\
\text { percutaneous } \\
\text { CVAD }\end{array}$ & 3 & 5 & - & 2 & - & 10 \\
\hline $\begin{array}{l}\text { Hemodialysis } \\
\text { catheters }\end{array}$ & - & - & - & 4 & 4 & 8 \\
\hline $\begin{array}{l}\text { Tunneled, } \\
\text { partially } \\
\text { implanted } \\
\text { CVAD }\end{array}$ & - & 1 & 13 & 5 & 1 & 20 \\
\hline $\begin{array}{l}\text { Totally } \\
\text { implantable } \\
\text { CVAD }\end{array}$ & - & - & 19 & 4 & 1 & 24 \\
\hline TOTAL & 24 & 6 & 38 & 24 & 9 & \\
\hline
\end{tabular}

\section{Study qualities}

The quality of the studies was mixed, with incomplete reporting of denominators of outcomes and poor outcome definition consistency to benchmarked standards evident. Twelve studies [59, 61, 66, 67, 72, 76, 82, 83, 89, 97, 105, 121] described the outcome of CVAD-associated BSI without the 
clarity and rigor of benchmarked standards, which meant that their CVAD-associated BSI data were not included within the review. Two studies [30,116] provided a combined 'all type' infection or mechanical failure outcomes, instead of providing separated local and systemic infection, occlusion and dislodgement data. These data were also not included in the review. We were unable to ascertain the number of catheter days in 23 studies, [66-82, 84, 85, 92, 97, 99, 122] which excluded their data from contributing to the meta-analysis reporting failure and complications per 1,000 catheter days.

\section{Synthesis of results}

\section{CVAD failure}

Table 2 reports the overall pooled proportion and IR of CVAD failure and complications across CVADs type. Figure 2 and 3 (Supplementary) report the individual and pooled proportion and IR of CVAD failure across CVADs type. Overall 25\% (95\% CI 20.9\%-29.2\%) of pediatric CVADs failed prior to completion of therapy; with an IR of 1.97 per 1,000 catheter days (95\% CI 1.71-2.23; 60 studies; 16,859 CVAD; 1,282,332 catheter days). Overall heterogeneity of studies reporting proportions of failure was high $\left(\mathrm{I}^{2}=97.1 \%\right)$, and moderate to high $\left(\mathrm{I}^{2}=75.0 \%-97.3 \%\right)$ when examined per device group. Umbilical catheters had the lowest pooled proportion of CVAD failure (11\%; 95\% CI 0.7\%-30.2\%) and the highest pooled IR per 1,000 catheter days (28.6; 95\% CI 17.439.8) with two and one (respectively) studies (426 CVADs; 979 catheter days) contributing to the analysis. HD catheters had the highest pooled proportion of CVAD failure (46.4\%; 95\% CI 29.6\%63.6\%; 323 CVAD). PICCs had the second-highest pooled IR per 1,000 catheter days (12.4; 95\% CI 10.0-14.9; 241,019 catheter days). Totally implanted devices had the lowest pooled IR of failure per 1,000 catheter days (0.15; 95\% CI 0.09-0.20; 819,022 catheter days). Because of a lack of data, a rate of CVAD failure per 1,000 catheter days for non-tunneled, percutaneous CVADs could not be estimated. 


\begin{tabular}{|c|c|c|c|c|c|c|c|c|c|c|c|}
\hline & \multirow[b]{2}{*}{ CVAD type } & \multicolumn{5}{|c|}{ Proportion of complications } & \multicolumn{5}{|c|}{ Incidence rates of complications / 1,000 catheter days } \\
\hline & & Studies & CVAD & Outcomes & Pooled \% & $95 \% \mathrm{CI}$ & Studies & $\begin{array}{l}\text { Catheter } \\
\text { days }\end{array}$ & Outcomes & Pooled IR & $95 \% \mathrm{CI}$ \\
\hline \multirow[t]{7}{*}{ Failure } & All & 60 & 16,859 & 4,121 & $\mathbf{2 5 . 0} \%(\mathrm{H}, \mathrm{S}, \mathrm{S})$ & $20.9-29.2$ & 37 & $1,282,332$ & 2,370 & $1.97(\mathrm{H}, \mathrm{S}, \mathrm{S})$ & $1.71-2.23$ \\
\hline & PICC & 23 & 10,163 & 2,771 & $30.1 \%(\mathrm{H}, \mathrm{S},-)$ & $24.4-36.1$ & 17 & 241,019 & 2,006 & $12.43(\mathrm{H}, \mathrm{S},-)$ & $9.98-14.89$ \\
\hline & Umbilical & 2 & 426 & 41 & $11.0 \%(\mathrm{H}, \mathrm{S},-)$ & $0.7-30.2$ & 1 & 979 & 28 & $28.60(-, \mathrm{S},-)$ & $17.44-39.77$ \\
\hline & Non-tunneled. & 2 & 1,126 & 248 & $16.7 \%(\mathrm{M}, \mathrm{S},-)$ & $6.1-30.9$ & 0 & - & - & - & - \\
\hline & $\mathrm{HD}$ & 4 & 323 & 124 & $46.4 \%(\mathrm{H}, \mathrm{S},-)$ & $29.6-63.6$ & 2 & 53,828 & 86 & $1.57(\mathrm{~L}, \mathrm{~S},-)$ & $1.16-1.99$ \\
\hline & Tunneled & 10 & 1,501 & 424 & $29.2 \%(\mathrm{H}, \mathrm{S},-)$ & $15.9-44.6$ & 6 & 167,484 & 123 & $0.86(\mathrm{H}, \mathrm{S},-)$ & $0.41-1.32$ \\
\hline & Totally implanted & 19 & 3,320 & 493 & $15.8 \%(\mathrm{H}, \mathrm{S},-)$ & $9.4-23.5$ & 10 & 819,022 & 127 & $0.15(\mathrm{M}, \mathrm{S},-)$ & $0.09-0.20$ \\
\hline \multirow{7}{*}{$\begin{array}{l}\text { CVAD- } \\
\text { associated } \\
\text { BSI }\end{array}$} & All & 75 & 31,933 & 2,899 & $10.3 \%(\mathrm{H}, \mathrm{S}, \mathrm{N})$ & $8.9-11.6$ & 50 & $1,338,756$ & 2,164 & $1.63(\mathrm{H}, \mathrm{S}, \mathrm{S})$ & $1.40-1.86$ \\
\hline & PICC & 27 & 16,428 & 1,081 & $8.6 \%(\mathrm{H}, \mathrm{S},-)$ & $7.0-10.2$ & 22 & 363,208 & 861 & $3.06(\mathrm{H}, \mathrm{S},-)$ & $2.39-3.72$ \\
\hline & Umbilical & 6 & 498 & 60 & $8.7 \%(\mathrm{H}, \mathrm{S},-)$ & $1.5-15.9$ & 1 & 979 & 33 & $33.71(-, \mathrm{S},-)$ & $21.64-45.78$ \\
\hline & Non-tunneled. & 10 & 11,020 & 1,028 & $8.7 \%(\mathrm{H}, \mathrm{S},-)$ & $3.6-13.8$ & 7 & 144,885 & 887 & $5.86(\mathrm{H}, \mathrm{S},-)$ & $3.38-8.34$ \\
\hline & $\mathrm{HD}$ & 4 & 323 & 31 & $10.4 \%(\mathrm{~L}, \mathrm{~S}-\mathrm{z})$ & $3.7-17.0$ & 2 & 53,828 & 23 & $0.41(\mathrm{~N}, \mathrm{~S},-)$ & $0.22-0.60$ \\
\hline & Tunneled. & 13 & 1,992 & 413 & $19.9 \%(\mathrm{H}, \mathrm{S},-)$ & $12.6-27.2$ & 8 & 188,807 & 189 & $1.13(\mathrm{H}, \mathrm{S},-)$ & $0.65-1.61$ \\
\hline & Totally implanted & 15 & 1,672 & 286 & $15.9 \%(\mathrm{H}, \mathrm{S},-)$ & $10.2-21.7$ & 10 & 587,049 & 171 & $0.28(\mathrm{H}, \mathrm{S},-)$ & $0.14-0.42$ \\
\hline Throm- & All & 53 & 15,979 & 471 & $1.7 \%(\mathrm{H}, \mathrm{S}, \mathrm{N})$ & $0.8-2.8$ & 30 & $1,168,248$ & 153 & $0.08(\mathrm{M}, \mathrm{S}, \mathrm{N})$ & $0.04-0.11$ \\
\hline \multirow[t]{6}{*}{ bosia } & PICC & 16 & 8,482 & 317 & $2.1 \%(\mathrm{H}, \mathrm{S},-)$ & $0.5-4.7$ & 12 & 226,931 & 61 & $0.17(\mathrm{M}, \mathrm{S},-)$ & $0.06-0.29$ \\
\hline & Umbilical & 3 & 402 & 12 & $3.7 \%(\mathrm{H}, \mathrm{N},-)$ & $0.0-12.2$ & 0 & - & - & - & - \\
\hline & Non-tynneled. & 4 & 1,370 & 23 & $3.7 \%(\mathrm{H}, \mathrm{S},-)$ & $0.0-11.1$ & 2 & 7,689 & 13 & $9.06\left(\mathrm{H}_{z} \mathrm{~N},-\right)$ & $0.00-28.4$ \\
\hline & $\mathrm{HD}$ & 3 & 264 & 7 & $2.9 \%(\mathrm{H}, \mathrm{N},-)$ & $0.0-12.8$ & 1 & 30,936 & 2 & $0.07(-, \mathrm{N},-)$ & $0.00-0.18$ \\
\hline & Tunneled. & 12 & 3,019 & 42 & $0.6 \%(\mathrm{~L}, \mathrm{~S}-)$ & $0.2-1.2$ & 7 & 335,689 & 20 & $0.04(\mathrm{~L}, \mathrm{~S},-)$ & $0.01-0.07$ \\
\hline & Totally implanted & 15 & 2,442 & 70 & $1.9 \%(\mathrm{H}, \mathrm{S},-)$ & $0.1-4.9$ & 8 & 567,003 & 57 & $0.06(\mathrm{H}, \mathrm{S},-)$ & $0.01-0.12$ \\
\hline Occlusion & All & 53 & 15,344 & 1,321 & $7.4 \%(\mathrm{H}, \mathrm{S}, \mathrm{N})$ & $5.5-9.6$ & 32 & 698,836 & 823 & $1.06(\mathrm{H}, \mathrm{S}, \mathrm{S})$ & $0.85-1.27$ \\
\hline \multirow[t]{6}{*}{ / blockage } & PICC & 23 & 9,786 & 837 & $8.2 \%(\mathrm{H}, \mathrm{S},-)$ & $5.9-10.9$ & 18 & 269,774 & 543 & $2.21(\mathrm{H}, \mathrm{S},-)$ & $1.66-2.77$ \\
\hline & Umbilical & 3 & 472 & 2 & $0.2 \%(\mathrm{~N}, \mathrm{~N},-)$ & $0.0-1.2$ & 1 & 979 & 0 & $0.00(-, N,-)$ & $0.00-1.88$ \\
\hline & Non-tunneled & 2 & 1,126 & 118 & $8.0 \%(\mathrm{M}, \mathrm{S},-)$ & $2.7-15.5$ & 0 & - & - & - & - \\
\hline & $\mathrm{HD}$ & 2 & 233 & 17 & $11.1 \%(\mathrm{H}, \mathrm{S},-)$ & $0.0-34.8$ & 1 & 30,936 & 8 & $0.26(\mathrm{H}, \mathrm{S},-)$ & $0.06-0.46$ \\
\hline & Tunneled. & 10 & 1,485 & 227 & $12.1 \%(\mathrm{H}, \mathrm{S},-)$ & $0.4-23.5$ & 7 & 280,516 & 216 & $0.85(\mathrm{H}, \mathrm{S},-)$ & $0.48-1.23$ \\
\hline & Totally implanted & 13 & 2,242 & 120 & $5.0 \%\left(\mathrm{H}, \mathrm{S}_{3}-\right)$ & $1.5-9.9$ & 5 & 116,631 & 56 & $0.30(\mathrm{H}, \mathrm{S},-)$ & $0.04-0.57$ \\
\hline
\end{tabular}

\begin{tabular}{|c|c|c|c|c|c|c|c|c|c|c|c|}
\hline Dislodge- & All & 39 & 9,784 & 686 & $4.7 \%(\mathrm{H}, \mathrm{S}, \mathrm{N})$ & $3.2-6.4$ & 23 & 645,611 & 437 & $0.43(\mathrm{H}, \mathrm{S}, \mathrm{S})$ & $0.30-0.56$ \\
\hline ment / & $\mathrm{PICC}$ & 14 & 5,389 & 389 & $5.4 \%(\mathrm{H}, \mathrm{S},-)$ & $3.3-8.0$ & 11 & 203,619 & 383 & $1.42(\mathrm{H}, \mathrm{S}-)$ & $0.70-2.14$ \\
\hline \multirow[t]{5}{*}{ migration } & Umbilical & 1 & 140 & 4 & $2.9 \%(-, \mathrm{S},-)$ & $0.6-6.4$ & 1 & 979 & 4 & $4.09(-, \mathrm{S},-)$ & $0.0-8.76$ \\
\hline & Non-tunneled & 2 & 1,126 & 91 & $3.5 \%(\mathrm{H}, \mathrm{N},-)$ & $0.0-15.2$ & 0 & - & - & - & - \\
\hline & $\mathrm{HD}$ & 3 & 264 & 14 & $8.8 \%(\mathrm{H}, \mathrm{S},-)$ & $0.1-26.0$ & 1 & 30,936 & 3 & $0.10(-, \mathrm{N}-)$ & $0.00-0.23$ \\
\hline & Tunneled. & 8 & 963 & 89 & $7.0 \%(\mathrm{H}, \mathrm{S},-)$ & $1.7-15.0$ & 5 & 154,725 & 41 & $0.24(\mathrm{H}, \mathrm{S}-)$ & $0.03-0.46$ \\
\hline & Totally implanted & 11 & 1,902 & 99 & $2.0 \%(\mathrm{H}, \mathrm{S},-)$ & $0.1-5.2$ & 5 & 256,962 & 6 & $0.02(\mathrm{~N}, \mathrm{~N},-)$ & $0.00-0.04$ \\
\hline Breakage / & All & 45 & 12,092 & 313 & $1.6 \%(\mathrm{H}, \mathrm{S}, \mathrm{S})$ & $0.9-2.5$ & 29 & 841,359 & 240 & $0.14(\mathrm{H}, \mathrm{S}, \mathrm{S})$ & $0.08-0.19$ \\
\hline \multirow[t]{6}{*}{ rupture } & $\mathrm{PICC}$ & 19 & 8,154 & 279 & $3.9 \%(\mathrm{H}, \mathrm{S},-)$ & $2.5-5.5$ & 15 & 226,990 & 218 & $0.88(\mathrm{H}, \mathrm{S},-)$ & $0.51-1.26$ \\
\hline & Umbilical & 1 & 140 & 0 & $0.0 \%(-, \mathrm{N},-)$ & $0.0-1.2$ & 1 & 979 & 0 & $0.00(-, \mathrm{N},-)$ & $0.00-1.88$ \\
\hline & Non-tunneled & 1 & 34 & 3 & $8.8 \%(-, \mathrm{S},-)$ & $1.2-21.2$ & 0 & - & - & - & - \\
\hline & $\mathrm{HD}$ & 3 & 264 & 2 & $0.5 \%(\mathrm{M}, \mathrm{N},-)$ & $0.0-5.3$ & 1 & 30,936 & 0 & $0.00(-, N,-)$ & $0.00-0.06$ \\
\hline & Tunneled & 8 & 963 & 21 & $1.1 \%(\mathrm{M}, \mathrm{S},-)$ & $0.0-3.1$ & 5 & 154,725 & 18 & $0.08(\mathrm{M}, \mathrm{N},-)$ & $0.00-0.17$ \\
\hline & Totally implanted & 13 & 2,179 & 8 & $0.0 \%(\mathrm{~N}, \mathrm{~N},-)$ & $0.0-0.0$ & 7 & 427,729 & 4 & $0.01(\mathrm{~N}, \mathrm{~N},-)$ & $0.00-0.02$ \\
\hline Local & All & 39 & 8,217 & 404 & $3.1 \%(\mathrm{H}, \mathrm{S}, \mathrm{S})$ & $2.0-4.4$ & 28 & 900,192 & 304 & $0.19(\mathrm{H}, \mathrm{S}, \mathrm{S})$ & $0.12-0.26$ \\
\hline infection / & PICC & 15 & 4,191 & 220 & $4.5 \%(\mathrm{M}, \mathrm{S},-)$ & $3.3-5.8$ & 13 & 176,590 & 219 & $1.32(\mathrm{H}, \mathrm{S},-)$ & $0.85-1.79$ \\
\hline \multirow[t]{5}{*}{ phlebitis } & Umbilical & 1 & 140 & 9 & $6.4 \%(-, \mathrm{S},-)$ & $2.9-11.2$ & 1 & 979 & 9 & $9.19(-, \mathrm{S},-)$ & $2.57-15.82$ \\
\hline & Non-tunneled & 2 & 259 & 0 & $0.0 \%(\mathrm{~L}, \mathrm{~N},-)$ & $0.0-0.4$ & 1 & 7,303 & 0 & $0.00(-, N,-)$ & $0.00-0.25$ \\
\hline & $\mathrm{HD}$ & 2 & 233 & 3 & $0.9 \%\left(\mathrm{~N}, \mathrm{~N}_{2}-\right)$ & $0.0-2.9$ & 1 & 30,936 & 3 & $0.10(-, \mathrm{N},-)$ & $0.00-0.23$ \\
\hline & Tunneled & 9 & 1,827 & 113 & $4.8 \%(\mathrm{H}, \mathrm{S},-)$ & $1.4-9.6$ & 5 & 154,725 & 64 & $0.38(\mathrm{H}, \mathrm{S},-)$ & $0.10-0.65$ \\
\hline & Totally implanted & 10 & 1,567 & 59 & $1.5 \%(\mathrm{H}, \mathrm{S},-)$ & $0.0-4.5$ & 7 & 529,659 & 9 & $0.01(\mathrm{~N}, \mathrm{~N},-)$ & $0.00-0.02$ \\
\hline
\end{tabular}

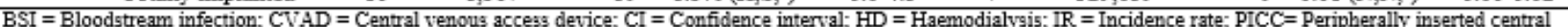
catheter, (H,S,S) etc.: first character describes heterogeneity of studies as negligible $(\mathrm{N})$, low $(\mathrm{L})$, moderate $(\mathrm{M})$ or high $(\mathrm{H})$, second describes result of effect-size test ( $\mathrm{S}=$ significant, $\mathrm{N}=$ non-significant), third describes result of test for heterogeneity between sub-groups, where applicable ( $\mathrm{S}=$ significant, $\mathrm{N}=$ non-significant);

\section{CVAD complications}

Table 2, Figures 4 and 5 (Supplementary) report the individual and pooled proportion and IR per 1,000 catheter days of CVAD-associated BSI, stratified by CVADs type. Overall, $10.3 \%$ (95\% CI 8.9\%-11.6\%; I²=95.0\%; 75 studies; 31,933 CVAD) of pediatric CVADs developed a CVADassociated BSI with an IR of 1.63 per 1,000 catheter days (95\% CI 1.40-1.86; I²=97.3\%; 50 studies; 
1,338,756 catheter days). Tunneled CVADs had the highest pooled proportion of CVAD-associated BSI (19.9\%; 95\% CI 12.6\%-27.2\%; 1,992 CVAD); while umbilical catheters had the highest pooled IR per 1,000 catheter days (IR 33.7; 95\% CI 21.6-45.8; 979 catheter days).

Tunneled CVADs had the highest pooled proportion of occlusion or blockage (12.1\%; 95\% CI 0.4\%-23.5\%; 1,485 CVAD) but PICCs had the highest pooled IR per 1,000 catheter days (2.2; 95\% CI 1.7-2.8; 269,774 catheter days). Tunneled CVADs had the highest proportion of local infection or phlebitis (4.8\%; 95\% CI 1.4-9.6; 1,827 catheter days), with umbilical catheters having the highest IR per 1,000 catheter days (9.2; 95\% CI 2.6-15.8; 979 catheter days). Totally implanted devices had the lowest proportion and rate of dislodgement per 1,000 catheter days for dislodgement (2.0\%, 95\% CI 0.1-5.1, 1,902 CVAD; 0.02, 95\%CI 0.00-0.04; 256,962 catheter days), and the lowest proportion of breakage / rupture (0.0\%, 95\%CI 0.0-0.0; 2,179 CVAD).

\section{Subgroup analyses}

The results of the subgroup analyses describing the pooled proportion and IR of CVAD failure per 1,000 catheter days across study populations are shown in Table 3. Due to availability of data, subgroup analyses were only carried out on PICCs (neonates and pediatrics; oncology/hematology and all others; outpatients and inpatients), tunneled CVADs (oncology/hematology and all others; outpatients and inpatients) and totally-implantable CVADs (oncology/hematology and all others). The rate of PICC failure per 1,000 catheter days was significantly $(\mathrm{P}<0.001)$ higher for neonates (IR 25.9; 95\% CI 21.2-30.5) compared with pediatric patients (IR 5.6; 95\% CI 3.2-8.1). PICCs managed in outpatient facilities had significantly $(\mathrm{P}=0.007)$ lower proportion of failure $(24.5 \%$; 95\% CI 16.9-32.8) in comparison to inpatient facilities (35.1\%; 95\% CI 27.3-43.2). Comparatively tunneled CVADs which were managed in outpatients facilities had significantly $(\mathrm{P}=0.016)$ higher proportion of failure (37.8\%; 95\% CI 17.0-61.3) in comparison to inpatient facilities (12.6\%; 95\% CI 8.5-17.3). 
Table 3: Subgroup analyses: Proportions and incidence rates of CVAD failure per device across study populations

\begin{tabular}{|c|c|c|c|c|c|c|c|c|c|c|c|}
\hline \multirow{2}{*}{$\begin{array}{c}\text { CVAD } \\
\text { type }\end{array}$} & \multirow{2}{*}{$\begin{array}{c}\text { Stady } \\
\text { population }\end{array}$} & \multicolumn{5}{|c|}{ Proportion of failure } & \multicolumn{5}{|c|}{ Incidence rates of failure per 1,000 cath. days } \\
\hline & & Studies & CVAD & Outcomes & Pooled \% & $95 \% \mathrm{CI}$ & Studies & $\begin{array}{l}\text { Catheter } \\
\text { days }\end{array}$ & Outcomes & $\begin{array}{c}\text { Pooled } \\
\text { incidence rates }\end{array}$ & $95 \% \mathrm{CI}$ \\
\hline \multirow[t]{9}{*}{ PICC } & Pooled & 22 & 8,596 & 2,346 & $30.2 \%(\mathrm{H}, \mathrm{S}, \mathrm{N})$ & $23.9-36.9$ & 17 & 176,568 & 1,581 & $13.06(\mathrm{H}, \mathrm{S}, \mathrm{S})$ & $10.39-15.73$ \\
\hline & Neonates & 11 & 4,521 & 1,472 & $35.4 \%(\mathrm{H}, \mathrm{S},-)$ & $25.0-46.6$ & 8 & 29,744 & 700 & $25.87(\mathrm{H}, \mathrm{S},-)$ & $21.23-30.52$ \\
\hline & Paediatrics & 11 & 4,138 & 894 & $25.0 \%(\mathrm{H}, \mathrm{S},-)$ & $18.2-32.5$ & 9 & 146,824 & 881 & $5.64(\mathrm{H}, \mathrm{S},-)$ & $3.18-8.09$ \\
\hline & Pooled & 23 & 10,163 & 2,791 & $30.1 \%(\mathrm{H}, \mathrm{S}, \mathrm{N})$ & $24.4-36.1$ & 18 & 241,019 & 2,006 & $12.43(\mathrm{H}, \mathrm{S}, \mathrm{N})$ & $9.98-14.89$ \\
\hline & Qnchaem & 4 & 357 & 119 & $30.0 \%(\mathrm{H}, \mathrm{S},-)$ & $15.2-47.3$ & 3 & 54,285 & 114 & $2.54(\mathrm{H}, \mathrm{S},-)$ & $0.55-4.53$ \\
\hline & All others & 19 & 9,743 & 2,652 & $30.1 \%(\mathrm{H}, \mathrm{S},-)$ & $23.9-36.7$ & 15 & 186,734 & 1,892 & $15.80(\mathrm{H}, \mathrm{S},-)$ & $12.44-19.15$ \\
\hline & Pooled & 51 & 10,072 & 2,783 & $31.3 \%(\mathrm{H}, \mathrm{S}, \mathrm{N})$ & $25.4-37.4$ & 18 & 241,019 & 2,006 & $12.43(\mathrm{H}, \mathrm{S}, \mathrm{S})$ & 9.98-14.89 \\
\hline & Outpatients & 32 & 3,673 & 748 & $24.5 \%(\mathrm{H}, \mathrm{S},-)$ & $16.9-32.8$ & 7 & 128,133 & 743 & $5.04(\mathrm{H}, \mathrm{S},-)$ & $2.28-7.80$ \\
\hline & Inpatients & 19 & 6,399 & 2,035 & $35.1 \%(\mathrm{H}, \mathrm{S},-)$ & $27.3-43.2$ & 11 & 112,886 & 1,263 & $19.91(\mathrm{H}, \mathrm{S},-)$ & $15.15-24.67$ \\
\hline \multirow[t]{6}{*}{ Tunneled } & Pooled & 11 & 1,501 & 424 & $29.2 \%(\mathrm{H}, \mathrm{S}, \mathrm{N})$ & $15.9-44.6$ & 6 & 167,484 & 123 & $0.87(\mathrm{H}, \mathrm{S}, \mathrm{N})$ & $0.41-1.32$ \\
\hline & Onchaem & 8 & 1,410 & 395 & $31.1 \%(\mathrm{H}, \mathrm{S},-)$ & $15.8-48.9$ & 5 & 166,068 & 122 & $0.88(\mathrm{H}, \mathrm{S},-)$ & $0.41-1.34$ \\
\hline & All others & 2 & 91 & 29 & $21.3 \%(\mathrm{H}, \mathrm{S},-)$ & $0.3-57.5$ & 1 & 1,416 & 1 & $0.71\left(-, \mathrm{N}_{2}-\right)$ & $0.00-2.67$ \\
\hline & Pooled & 7 & 1,281 & 375 & $33.6 \%(\mathrm{H}, \mathrm{S}, \mathrm{S})$ & $16.1-53.8$ & 4 & 146,740 & 102 & $0.84(\mathrm{H}, \mathrm{S}, \mathrm{N})$ & $0.32-1.35$ \\
\hline & Outpatients & 6 & 1,058 & 347 & $37.8 \%(\mathrm{H}, \mathrm{S},-)$ & $17.0-61.3$ & 3 & 94,901 & 74 & $1.20(\mathrm{H}, \mathrm{S},-)$ & $0.30-2.10$ \\
\hline & Inpatients & 1 & 223 & 28 & $12.6 \%\left(-, \mathrm{S}_{2}-\right)$ & $8.5-17.3$ & 1 & 51,839 & 28 & $0.54(-, \mathrm{S},-)$ & $0.33-0.75$ \\
\hline Totally & Pooled & 18 & 3,320 & 493 & $15.8 \%(\mathrm{H}, \mathrm{S}, \mathrm{N})$ & $9.4-23.5$ & 10 & 819,022 & 127 & $0.15(\mathrm{M}, \mathrm{S},-)$ & $0.09-0.20$ \\
\hline implant- & Qnchaem & 17 & 2,694 & 361 & $15.2 \%(\mathrm{H}, \mathrm{S},-)$ & $8.1-23.9$ & 10 & 819,022 & 127 & $0.15(\mathrm{M}, \mathrm{S},-)$ & $0.09-0.20$ \\
\hline ted & All others & 2 & 626 & 132 & $21.1 \%(\mathrm{~L}, \mathrm{~S},-)$ & $17.9-24.4$ & 0 & - & - & - & - \\
\hline
\end{tabular}

\section{Sensitivity analyses}

Table 4 describes the results of sensitivity analyses comparing pooled proportion and IR of CVAD

failure across study methods. The majority of studies reporting CVAD failure were completed using retrospective methods (73.6\% studies; 64.8\% CVAD; 78.9\% catheter days). Overall IRs of failure were not different between prospective and retrospective studies. There was significant difference between study types in reported proportions of failure of non-tunneled devices (prospective: 22.4\%; 95\% CI 20.0-25.0; retrospective: 8.8\%; 95\% CI 1.2-21.2; $\mathrm{p}=0.046$ ). Sensitivity analyses could not be undertaken for HD catheters as all included studies used retrospective study methods.

Table 4: Sensitivity analyses: Proportions and incidence rates of CVAD failure per device reported in prospective and retrospective studies

\begin{tabular}{|c|c|c|c|c|c|c|c|c|c|c|c|}
\hline \multirow{2}{*}{$\underset{\text { type }}{\text { CVAD }}$} & \multirow{2}{*}{$\begin{array}{l}\text { Study } \\
\text { method }\end{array}$} & \multicolumn{5}{|c|}{ Proportion of failure } & \multicolumn{5}{|c|}{ Incidence rate of failure per 1,000 cath. days } \\
\hline & & Studies & CVAD & Outcomes & Pooled \% & $95 \% \mathrm{CI}$ & Studies & $\begin{array}{l}\text { Catheter } \\
\text { days }\end{array}$ & Outcomes & $\begin{array}{c}\text { Pooled } \\
\text { incidence rate }\end{array}$ & $95 \% \mathrm{CI}$ \\
\hline \multirow[t]{3}{*}{ PICC } & Pooled & 23 & 10,163 & 2,791 & $30.1 \%(\mathrm{H}, \mathrm{S}, \mathrm{N})$ & $24.4-36.1$ & 18 & 241,019 & 2,006 & $12.43(\mathrm{H}, \mathrm{S}, \mathrm{N})$ & $9.98-14.89$ \\
\hline & Retrospect. & 17 & 6,609 & 1,876 & $28.3 \%(\mathrm{H}, \mathrm{S},-)$ & $21.1-36.1$ & 14 & 157,350 & 1,272 & $12.49(\mathrm{H}, \mathrm{S},-)$ & $9.69-15.30$ \\
\hline & Prospective & 6 & 3,554 & 915 & $35.1 \%(\mathrm{H}, \mathrm{S},-)$ & $23.8-47.4$ & 4 & 83,669 & 734 & $13.17(\mathrm{H}, \mathrm{S},-)$ & $5.10-21.25$ \\
\hline \multirow{3}{*}{$\begin{array}{l}\text { Non- } \\
\text { tumneled }\end{array}$} & Pooled & 2 & 1,126 & 248 & $16.7 \%(\mathrm{M}, \mathrm{S}, \mathrm{S})$ & $6.1-30.9$ & 0 & - & - & - & - \\
\hline & Retrospect. & 1 & 34 & 3 & $8.8 \%(-, S,-)$ & $1.2-21.2$ & 0 & - & - & - & - \\
\hline & Prospective & 1 & 1,092 & 245 & $22.4 \%\left(-, \mathrm{S}_{2}-\right)$ & $20.0-25.0$ & 0 & - & - & - & - \\
\hline \multirow[t]{3}{*}{ Tunneled } & Pooled & 10 & 1,501 & 424 & $29.2 \%(\mathrm{H}, \mathrm{S}, \mathrm{N})$ & $15.9-44.6$ & 6 & 167,484 & 123 & $0.87(\mathrm{H}, \mathrm{S}, \mathrm{N})$ & $0.41-1.32$ \\
\hline & Retrospect. & 7 & 1,110 & 291 & $30.6 \%(\mathrm{H}, \mathrm{S},-)$ & $15.4-48.4$ & 4 & 129,538 & 96 & $1.08(\mathrm{H}, \mathrm{S},-)$ & $0.40-1.75$ \\
\hline & Prospective & 3 & 391 & 133 & $26.1 \%(\mathrm{H}, \mathrm{S},-)$ & $1.6-65.0$ & 2 & 37,946 & 27 & $0.68(\mathrm{H}, \mathrm{S},-)$ & $0.04-1.32$ \\
\hline Totally & Pooled & 19 & 3,320 & 493 & $15.8 \%(\mathrm{H}, \mathrm{S}, \mathrm{N})$ & $9.4-23.5$ & 10 & 819,022 & 127 & $0.15(\mathrm{M}, \mathrm{S}, \mathrm{N})$ & $0.09-0.20$ \\
\hline implant- & Retrospect. & 14 & 2,547 & 420 & $15.0 \%(\mathrm{H}, \mathrm{S},-)$ & $9.9-20.9$ & 8 & 681,196 & 117 & $0.17(\mathrm{M}, \mathrm{S},-)$ & $0.10-0.24$ \\
\hline & Prospective & 5 & 773 & 73 & $18.7 \%(\mathrm{H}, \mathrm{S},-)$ & $0.0-54.7$ & 2 & 137,826 & 10 & $0.06(\mathrm{~N}, \mathrm{~S},-)$ & $0.02-0.11$ \\
\hline
\end{tabular}

Retrospect $=$ retrospective $;$ CVAD = Central venous access device; $\mathrm{CI}=$ Confidence interval; $\mathrm{IR}=$ Incidence rate; PICC= Peripherally inserted central catheter $(\mathrm{H}, \mathrm{S}, \mathrm{N})$ etc.: first character describes heterogeneity of studies as low $(\mathrm{L})$, moderate $(\mathrm{M})$ or high $(\mathrm{H}) /$ second describes result of effect-size test (S=significant, $\mathrm{N}=$ non-significant) / third describes result of test for heterogeneity between sub-groups, where applicable ( $\mathrm{S}=$ significant, $\mathrm{N}=$ non-significant);

\section{Discussion}


This study has, for the first time, carefully found, critiqued and synthesized CVAD failure rates, across CVAD types and pediatric populations. The results clearly show that failure of CVADs throughout pediatrics is a substantial and significant problem, with one in four failing. This is especially prevalent within the lifespan of umbilical catheters and PICCs. These devices have been traditionally recommended for short to medium term use, $[4,6]$ but the PICCs and umbilical catheters described within the included studies failed prior to the completion of therapy in $11-30 \%$ of cases, with a pooled incidence failure rate of 12-29 per 1,000 catheter days. This high rate of pediatric umbilical catheter and PICC failure is also evident in reported rates of catheter-associated BSI, occlusion, dislodgement and local infection/phlebitis. There is no previous umbilical catheter meta-analysis to benchmark these results and only small studies included within this review. However our results in PICCs and recent studies by Chopra and colleagues in adults [126, 127] have demonstrated that PICCs are substantially more problematic than originally thought. The outcomes of PICCs used in clinical practice needs to be cautiously and systematically monitored. Clinicians should be made aware of the high rates of failure associated with their use, and should question whether PICCs are the suitable intravascular device for their patient group. Research needs to be undertaken to discover and evaluate innovative strategies to reduce PICC and umbilical catheter failures, through examining insertion procedures, securement devices and patency practices.

Totally implanted devices were frequently associated with the lowest pooled incidence rate of failure and complications. These devices have previously been credited with improved ease of medication administration, decreased infectious risks and improved patient quality of life.[76] While the insertion of totally implanted and other tunneled CVAD requires the skills and resources of an experienced surgeon and operating theatre, fatal complications from cardiac tamponade and major vessel injury are rare.[10] It may be that due to lower rates of failure and complications, the cost-effectiveness of totally implanted devices is superior to other CVAD types for some population groups. Randomized controlled trials (RCTs) studying comparative clinical and cost-effectiveness Page $\mid 16$ 
of totally implanted devices, compared to other intravascular device types in suitable populations (e.g. cystic fibrosis) are urgently needed.

Given that many cases of CVAD failure and complications are thought to be avoidable,[6] the overall rate of CVAD failure and complication for children across all CVAD types appears variable, but remains unacceptably high. There are no current benchmarked targets for clinicians to compare their current rates of CVAD failure and complications, with the exception of CVAD-associated BSI in the ICU $[128,129]$. Quality improvement studies have previously demonstrated a marked reduction in complication rates associated with CVAD in pediatrics and neonates, indicating that complication rates are dependent upon the care provided by multidisciplinary clinicians.[130-132] Previous international focus on the prevention of CVAD complications from organizations such as the World Health Organization (WHO) and the Centers for Disease Control and Prevention (CDC) has been solely focused on CVAD-associated BSI, and generally in the ICU setting. However, our data demonstrates that there is also a high rate of failure due to occlusion, thrombosis, breakage and dislodgement. The prevalence of thrombosis is likely to be higher than described, as some included studies relied upon clinical suspicion of thrombosis, rather than routine imaging, significantly underestimating the true proportion/rate of CVAD-related thrombosis. These mechanical complications also result in an interruption to necessary treatment and the insertion of new CVAD, and should be the focus of the next generation of multidisciplinary international CVAD campaigns for improvement.

The subgroup analyses demonstrated the variation in CVAD failure based upon patient age. The variation was most evident in comparisons involving PICCs, where neonates had a significantly higher rate of failure $(\mathrm{p}<0.001)$, than the remaining pediatric population, with a failure rate of 25.5 per 1,000 catheter days. PICCs are extensively used to provide hyperosmolar solutions, inotropic medicines and parenteral nutrition within the neonatal period.[124] The neonates requiring PICCs are often very low birth weight (smaller than 1,500g at birth) or extremely low birth weight (smaller 
than 1,000 g at birth), and are at greatest risk for failure and its sequelae.[60, 117] The increasing use of PICCs within the neonatal population requires caution and careful surveillance, and should be the focus of significant innovation for improvement.

Our study has demonstrated current gaps in the breadth and quality of research into pediatric CVAD failure and complications. Further prospective cohort studies estimating the rates of failure and complications of CVAD in pediatrics are necessary to provide benchmarking targets and inform practice innovations. Meta-synthesis of the two studies reporting non-tunneled CVAD failure reported a failure proportion of $16.7 \%$, however no studies reported an estimation of catheter days. In accordance with previous international focus, the majority of non-tunneled CVAD studies which reported CVAD complications only reported CVAD-associated BSI. The failure and complications associated with HD and umbilical catheters were also inadequately reported, with only six studies of 749 CVADs available. Considering the prevalence and importance of umbilical, HD and nontunneled CVADs within pediatric healthcare management, reliable measurement of their failure and complications are essential. Additionally, while multiple cohort studies described the failure of PICCs and totally implanted devices, the majority used retrospective methods, using less reliable means of data collection. Future descriptions of CVAD failure need to be planned prospectively, use validated definitions for outcome measures and report denominator information including catheter days.

Our study results should be interpreted in the context of some limitations. Not all study authors were able to provide the total number of catheter days, which limited their data being included in the meta-analysis per 1,000 catheter days. Such time-based analysis is the more valid way to compare CVAD complication incidence, since the different dwell times typical of the CVAD types already exposes the patient to more or less risk of complications. Secondly, the unavoidable heterogeneity of the study populations in the included studies may have impacted on the generalizability of the results, subgroup analyses were used to reduce this problem. The levels of 
statistical heterogeneity of the final analyses are indicative of the heterogeneous group of pediatric patients which require CVADs. This heterogeneity needs to be recognized before applying the results to local individual healthcare institutions. Thirdly, while our review was limited to include studies up to ten years of age, many quality improvement activities have been instituted in pediatric facilities to prevent complications and failures associated with CVAD within that period. It is therefore possible that the pooled data may therefore overestimate the burden of device failure in 2015. Finally, while our study presents the association between CVAD-types and failure and complications, these results do not reflect causation. Without RCTs comparing the various CVAD types, it is impossible to assert that one CVAD type reduces complications and failure in comparison to another. Future updates of this review may also consider the inclusion of the ‘standard care’ arm of RCTs which evaluated various interventions.

\section{Comparison with other studies}

There has not been a previous systematic review undertaken in pediatrics examining the failure and complications associated with different CVADs. Landmark work by McGee and Gould [133] described the prevention, treatment and incidence of CVAD failure in the adult population. While primarily focused on describing strategies to prevent and treat CVAD complications, their systematic review reported an overall incidence of CVAD failure of greater than $15 \%$, with mechanical complications reported in $5 \%$ to $19 \%$ of patients, and infectious complications in 5 to 26\%. Our study describes a higher rate of mechanical and infectious complications which may be due to differing synthesis methodology and the underlying vulnerability and other clinical characteristics of the population studied.

\section{Conclusion and future research}


International healthcare institutions have highlighted the significance of CVAD failure associated with BSI. This systematic review has described the broader, multi-focal rate of CVAD failure and complications across CVAD type in pediatrics within the international healthcare community. Contextually, from the 82 US hospitals reporting to the National Healthcare Safety Network in 2013, greater than 2.7 million CVAD catheter days in the pediatric and neonatal population were registered. [134] Applying the rate of failure described in our study, 5,457 pediatric and neonatal CVADs in US hospitals failed prior to completion of treatment in one year alone. These failures place a massive economic and physical burden on the US healthcare system, patients and families.

Strategies have been developed to prevent CVAD failure by focusing on different aspects of infectious and mechanical complications, and their pathogenesis. The current rate of CVAD failure in pediatrics demonstrates that further evidence-based improvements to their insertion and maintenance are necessary. This includes insertion and maintenance practices surrounding CVAD dressing and securement, needleless access devices, flushing procedures and CVAD materials. Research is required urgently to develop and apply innovative and effective solutions to prevent CVAD failure in this vulnerable pediatric group.

Acknowledgements: Thank you to all the study authors who were able to contribute additional data.

Word count: 3,988/4,000 


\section{References:}

1. Carraro F, Cicalese M P, Cesaro S, et al., Guidelines for the use of long-term central venous catheter in children with hemato-oncological disorders. On behalf of supportive therapy working group of Italian Association of Pediatric Hematology and Oncology (AIEOP). Ann Hematol, 2013. 92(10): p. 1405-12.

2. Perdikaris P, Petsios K, Vasilatou-Kosmidis H, et al., Complications of Hickman-Broviac catheters in children with malignancies. Pediatr Hematol Oncol, 2008. 25(5): p. 375-84.

3. Cesaro S, Corro R, Pelosin A, et al., A prospective survey on incidence and outcome of Broviac/Hickman catheter-related complications in pediatric patients affected by hematological and oncological diseases. Ann Hematol, 2004. 83(3): p. 183-8.

4. Pittiruti M, Hamilton H, Biffi R, et al., ESPEN Guidelines on Parenteral Nutrition: central venous catheters (access, care, diagnosis and therapy of complications). Clin Nutr, 2009. 28(4): p. 365-77.

5. O'Grady N P, Alexander M, Burns L A, et al., Guidelines for the prevention of intravascular catheter-related infections. Clin Infect Dis, 2011. 52(9): p. e162-93.

6. Loveday H P, Wilson J A, Pratt R J, et al., epic3:National Evidence-based guidelines for preventing healthcare-associated infections. J Hosp Infect, 201486 (S1): p. S1-70.

7. Ruebner R, Keren R, Coffin S, et al., Complications of central venous catheters used for the treatment of acute hematogenous osteomyelitis. Pediatrics, 2006. 117(4): p. 1210-5.

8. Peng C, Monagle P, and Newall F, Clinical outcomes of management of CVAD occlusions. Arch Dis Child, 2011. 96(9): p. 885-7.

9. van Miert C, Hill R, and Jones L, Interventions for restoring patency of occluded central venous catheter lumens. Cochrane Database Syst Rev, 2012. 4: p. Cd007119.

10. Askegard-Giesmann J R, Caniano D A, and Kenney B D, Rare but serious complications of central line insertion. Semin Pediatr Surg, 2009. 18(2): p. 73-83.

11. Rey C, Alvarez F, De La Rua V, et al., Mechanical complications during central venous cannulations in pediatric patients. Intensive Care Med, 2009. 35(8): p. 1438-43.

12. Gibson F and Bodenham A, Misplaced central venous catheters: applied anatomy and practical management. Br J Anaesth, 2013. 110(3): p. 333-46.

13. Alexandrou E, Spencer T R, Frost S A, et al., Central venous catheter placement by advanced practice nurses demonstrates low procedural complication and infection rates--a report from 13 years of service. Crit Care Med, 2014. 42(3): p. 536-43.

14. Ullman A J, Long D A, and Rickard C M, Prevention of central venous catheter infections: a survey of paediatric ICU nurses' knowledge and practice. Nurse Educ Today, 2014. 34(2): p. 202-7.

15. Miller M R, Niedner M F, Huskins W C, et al., Reducing PICU Central Line-Associated Bloodstream Infections: 3-Year Results. Pediatrics, 2011. 128(5): p. e1077-e1083.

16. Ullman A J, Mitchell M, Lin F, et al., Dressings and securement devices for central venous catheters (CVC) (Review). Cochrane Database of Systematic Reviews 2015. accepted for publication.

17. Maki D G, Weise C E, and Sarafin H W, A semiquantitative culture method for identifying intravenous-catheter-related infection. $N$ Engl J Med, 1977. 296(23): p. 1305-1309.

18. Mermel L A, What is the predominant source of intravascular catheter infections? Clin Infect Dis, 2011. 52: p. 211-212.

19. Mermel, Allon M, Bouza E, et al., Clinical practice guidelines for the diagnosis and management of intravascular catheter-related infection: 2009 update by the Infectious Diseases Society of America. Clin Infect Dis, 2009. 49: p. 1-45.

20. Centers for Disease Control and Prevention, National Healthcare Safety Network Device Associated Module: CLABSI, U.S.o.A. Government, Editor. 2014: Atlanta. p. 1-9. 
21. Halton K A, Cook D, Paterson D L, et al., Cost-effectiveness of a central venous catheter care bundle. PLoS One, 2010. 5(9).

22. Cooper K, Frampton G, Harris P, et al., Are educational interventions to prevent catheterrelated bloodstream infections in intensive care unit cost-effective? J Hosp Infect, 2014. 86(1): p. 47-52.

23. Kim J S, Holtom P, and Vigen C, Reduction of catheter-related bloodstream infections through the use of a central venous line bundle: epidemiologic and economic consequences. Am J Infect Control, 2011. 39(8): p. 640-6.

24. Schwebel C, Lucet J C, Vesin A, et al., Economic evaluation of chlorhexidine-impregnated sponges for preventing catheter-related infections in critically ill adults in the dressing study. Crit Care Med, 2012. 40(1): p. 11-17.

25. Barnacle A, Arthurs O J, Roebuck D, et al., Malfunctioning central venous catheters in children: a diagnostic approach. Pediatr Radiol, 2008. 38(4): p. 363-78, quiz 486-7.

26. Stroup D F, Berlin J A, Morton S C, et al., Meta-analysis of observational studies in epidemiology: a proposal for reporting. Meta-analysis Of Observational Studies in Epidemiology (MOOSE) group. JAMA, 2000. 283(15): p. 2008-12.

27. Rickard C M, Webster J, Wallis M C, et al., Routine versus clinically indicated replacement of peripheral intravenous catheters: a randomised controlled equivalence trial. Lancet, 2012. 380(9847): p. 1066-74.

28. Prieto-Merino D, Smeeth L, Staa T P, et al., Dangers of non-specific composite outcome measures in clinical trials. BMJ, 2013. 347: p. f6782.

29. Schulz K F, Altman D F, Moher D, et al., CONSORT 2010 statement: updated guidelines for reporting parallel group randomised trials. Int J Surg, 2011. 9(8): p. 672-7.

30. Fratino G, Molinari A C, Parodi S, et al., Central venous catheter-related complications in children with oncological/hematological diseases: an observational study of 418 devices. Ann Oncol, 2005. 16(4): p. 648-54.

31. Nyaga V N, Abryn M, and Aerts M, Metaprop: a Stata command to perform meta-analysis of binomial data. Archive of Public Health, 2014. 72: p. 39.

32. StataCorp, Stata Statistical Software: Release 12.1. 201, StataCorp LP: College Station, TX.

33. Vandenbroucke J P, von Elm E, Altman D G, et al., Strengthening the Reporting of Observational Studies in Epidemiology (STROBE): Explanation and elaboration. Int J Surg, 2014.

34. Moher D, Liberati A, Tetzlaff J, et al., Preferred reporting items for systematic reviews and meta-analyses: the PRISMA statement. Int J Surg, 2010. 8(5): p. 336-41.

35. Dal Molin A, Di Massimo D S, Braggion C, et al., Totally implantable central venous access ports in patients with cystic fibrosis: a multicenter prospective cohort study. J Vasc Access, 2012. 13(3): p. 290-5.

36. Zaritsky J J, Salusky I B, Gales B, et al., Vascular access complications in long-term pediatric hemodialysis patients. Pediatr Nephrol, 2008. 23(11): p. 2061-5.

37. Dheer G, Chaudhry G K, and Singh T, Immediate complications of percutaneous central venous cannulation in children. J Indian Assoc Pediatr Surg, 2011. 16(4): p. 145-7.

38. Dzierzega M, Ossowska M, Chmiel D, et al., The malposition of central venous catheters in children. Pol J Radiol, 2014. 79: p. 275-8.

39. Malbezin S, Gauss T, Smith I, et al., A review of 5434 percutaneous pediatric central venous catheters inserted by anesthesiologists. Paediatr Anaesth, 2013. 23(11): p. 974-9.

40. Wiegering V, Schmid S, Andres O, et al., Thrombosis as a complication of central venous access in pediatric patients with malignancies: a 5-year single-center experience. BMC Hematol, 2014. 14(1): p. 18.

41. Fallon S C, Kim M E, Fernandes C J, et al., Identifying and reducing early complications of surgical central lines in infants and toddlers. J Surg Res, 2014. 190(1): p. 246-50. 
42. Haddad H, Lee K S, Higgins A, et al., Routine surveillance ultrasound for the management of central venous catheters in neonates. J Pediatr, 2014. 164(1): p. 118-22.

43. Robinson J L, Casey L M, Huynh H Q, et al., Prospective cohort study of the outcome of and risk factors for intravascular catheter-related bloodstream infections in children with intestinal failure. J Parenter Enteral Nutr, 2013.

44. Journeycake J M and Buchanan G R, Catheter-related deep venous thrombosis and other catheter complications in children with cancer. J Clin Oncol, 2006. 24(28): p. 4575-80.

45. Allen R C, Holdsworth M T, Johnson C A, et al., Risk determinants for catheter-associated blood stream infections in children and young adults with cancer. Pediatr Blood Cancer, 2008. 51(1): p. 53-8.

46. Blanchard A C, Fortin E, Rocher I, et al., Central line-associated bloodstream infection in neonatal intensive care units. Infect Control Hosp Epidemiol, 2013. 34(11): p. 1167-73.

47. McLean T W, Fisher C J, Snively B M, et al., Central venous lines in children with lesser risk acute lymphoblastic leukemia: optimal type and timing of placement. J Clin Oncol, 2005. 23(13): p. 3024-9.

48. Yang R Y, Moineddin R, Filipescu D, et al., Increased complexity and complications associated with multiple peripherally inserted central catheter insertions in children: the tip of the iceberg. J Vasc Interv Radiol, 2012. 23(3): p. 351-7.

49. Elihu A and Gollin G, Complications of implanted central venous catheters in neutropenic children. Am Surg, 2007. 73(10): p. 1079-82.

50. Colacchio K, Deng Y, Northrup V, et al., Complications associated with central and noncentral venous catheters in a neonatal intensive care unit. J Perinatol, 2012. 32(12): p. 9416.

51. Fadel F I, Abdel Mooty H N, Bazaraa H M, et al., Central venous catheters as a vascular access modality for pediatric hemodialysis. Int Urol Nephrol, 2008. 40(2): p. 489-96.

52. Hussain S, Gomez M M, Wludyka P, et al., Survival times and complications of catheters used for outpatient parenteral antibiotic therapy in children. Clin Pediatr (Phila), 2007. 46(3): p. 247-51.

53. Jain A, Deshpande P, and Shah P, Peripherally inserted central catheter tip position and risk of associated complications in neonates. J Perinatol, 2013. 33(4): p. 307-12.

54. Xia B, Tang J, Xiong Y, et al., Peripherally inserted central catheters and the incidence of candidal sepsis in VLBW and ELBW infants: is sepsis increased? World J Pediatr, 2010. 6(2): p. 154-7.

55. Revel-Vilk S, Yacobovich J, Tamary H, et al., Risk factors for central venous catheter thrombotic complications in children and adolescents with cancer. Cancer, 2010. 116(17): p. 4197-205.

56. Costa P, Bueno M, Alves A M, et al., Incidence of nonelective removal of percutaneously inserted central catheters according to tip position in neonates. J Obstet Gynecol Neonatal Nurs, 2013. 42(3): p. 348-56.

57. Yumani D F, van den Dungen F A, and van Weissenbruch M M, Incidence and risk factors for catheter-associated bloodstream infections in neonatal intensive care. Acta Paediatr, 2013. 102(7): p. e293-8.

58. Wagner M, Bonhoeffer J, Erb T O, et al., Prospective study on central venous line associated bloodstream infections. Arch Dis Child, 2011. 96(9): p. 827-31.

59. Uygun I, Okur M H, Otcu S, et al., Peripherally inserted central catheters in the neonatal period. Acta Cir Bras, 2011. 26(5): p. 404-11.

60. Ozkiraz S, Gokmen Z, Anuk Ince D, et al., Peripherally inserted central venous catheters in critically ill premature neonates. J Vasc Access, 2013. 14(4): p. 320-4.

61. Nam S H, Kim D Y, Kim S C, et al., Complications and risk factors of infection in pediatric hemato-oncology patients with totally implantable access ports (TIAPs). Pediatr Blood Cancer, 2010. 54(4): p. 546-51. 
62. Casner M, Hoesli S J, Slaughter J C, et al., Incidence of catheter-related bloodstream infections in neonates following removal of peripherally inserted central venous catheters. Pediatr Crit Care Med, 2014. 15(1): p. 42-8.

63. Barrier A, Williams D J, Connelly M, et al., Frequency of peripherally inserted central catheter complications in children. Pediatr Infect Dis J, 2012. 31(5): p. 519-21.

64. Aiyagari R, Song J Y, Donohue J E, et al., Central venous catheter-associated complications in infants with single ventricle: comparison of umbilical and femoral venous access routes. Pediatr Crit Care Med, 2012. 13(5): p. 549-53.

65. Chelliah A, Heydon K H, Zaoutis T E, et al., Observational trial of antibiotic-coated central venous catheters in critically ill pediatric patients. Pediatr Infect Dis J, 2007. 26(9): p. 81620.

66. White A D, Othman D, Dawrant M J, et al., Implantable versus cuffed external central venous catheters for the management of children and adolescents with acute lymphoblastic leukaemia. Pediatr Surg Int, 2012. 28(12): p. 1195-9.

67. Mangum D S, Verma A, Weng C, et al., A comparison of central lines in pediatric oncology patients: Early removal and patient centered outcomes. Pediatr Blood Cancer, 2013. 60(11): p. 1890-5.

68. Tercier S, Gapany C, Diezi M, et al., Incidents and complications of totally implanted vascular access devices in children: a prospective study. Patient Saf Surg, 2008. 2(1): p. 30.

69. Adler A, Yaniv I, Steinberg R, et al., Infectious complications of implantable ports and Hickman catheters in paediatric haematology-oncology patients. J Hosp Infect, 2006. 62(3): p. 358-65.

70. Almuneef M A, Memish Z A, Balkhy H H, et al., Rate, risk factors and outcomes of catheter-related bloodstream infection in a paediatric intensive care unit in Saudi Arabia. $J$ Hosp Infect, 2006. 62(2): p. 207-13.

71. Bezzio S, Scolfaro C, Broglia R, et al., Prospective incidence study of bloodstream infection in infants and children with central venous catheters after cardiac surgery in Italy. Infect Control Hosp Epidemiol, 2009. 30(7): p. 698-701.

72. Bratton J, Johnstone P A, and McMullen K P, Outpatient management of vascular access devices in children receiving radiotherapy: Complications and morbidity. Pediatr Blood Cancer, 2013.

73. Can E, Salihoglu O, Ozturk A, et al., Complication profiles of central and non-central $1 \mathrm{Fr}$ PICCs in neonates weighing <1500 g. J Matern Fetal Neonatal Med, 2014. 27(15): p. 15225.

74. Cheng H Y, Lu C Y, Huang L M, et al., Increased frequency of peripheral venipunctures raises the risk of central-line associated bloodstream infection in neonates with peripherally inserted central venous catheters. J Microbiol Immunol Infect, 2014.

75. Delarbre B, Dabadie A, Stremler-Lebel N, et al., Introduction of the use of a pediatric PICC line in a French University Hospital: review of the first 91 procedures. Diagn Interv Imaging, 2014. 95(3): p. 277-81.

76. Fallon S C, Larimer E L, Gwilliam N R, et al., Increased complication rates associated with Port-a-Cath placement in pediatric patients: location matters. J Pediatr Surg, 2013. 48(6): p. 1263-8.

77. Gapany C, Tercier S, Diezi M, et al., Frequent accesses to totally implanted vascular ports in pediatric oncology patients are associated with higher infection rates. J Vasc Access, 2011. 12(3): p. 207-10.

78. Lopez P J, Troncoso B, Grandy J, et al., Outcome of tunnelled central venous catheters used for haemodialysis in children weighing less than $15 \mathrm{~kg}$. J Pediatr Surg, 2014. 49(8): p. 1300-3.

79. Njere I, Islam S, Parish D, et al., Outcome of peripherally inserted central venous catheters in surgical and medical neonates. J Pediatr Surg, 2011. 46(5): p. 946-50. 
80. Peynircioglu B, Ozkan F, Canyigit M, et al., Radiologically placed tunneled internal jugular catheters in the management of chronic hemodialysis and long-term infusion therapies in the pediatric population. J Vasc Interv Radiol, 2007. 18(7): p. 875-81.

81. Pinon M, Bezzio S, Tovo P A, et al., A prospective 7-year survey on central venous catheter-related complications at a single pediatric hospital. Eur J Pediatr, 2009. 168(12): p. 1505-12.

82. Rouzrokh M, Shamsian B S, KhaleghNejad Tabari A, et al., Totally implantable subpectoral vs. subcutaneous port systems in children with malignant diseases. Arch Iran Med, 2009. 12(4): p. 389-94.

83. Sharp N E, Knott E M, Thomas P, et al., Burden of complications from needle penetration of plastic ports in children. J Pediatr Surg, 2014. 49(5): p. 763-5.

84. Smitherman A B and Weston B W, Catheter-associated thrombosis in children: singleinstitution experience and review of pediatric venous thromboembolic disease. J Infus Nurs, 2014. 37(2): p. 103-7.

85. Thornburg C D, Smith P B, Smithwick M L, et al., Association between thrombosis and bloodstream infection in neonates with peripherally inserted catheters. Thromb Res, 2008. 122(6): p. 782-5.

86. Albisetti M, Kellenberger C J, Bergstrasser E, et al., Port-a-cath-related thrombosis and postthrombotic syndrome in pediatric oncology patients. J Pediatr, 2013. 163(5): p. 1340-6.

87. Bartram J L, O'Driscoll S, Kulasekararaj A G, et al., Portacaths are safe for long-term regular blood transfusion in children with sickle cell anaemia. Arch Dis Child, 2011. 96(11): p. 1082-4.

88. Bucki B, Tomaszewska R, Karpe J, et al., Central venous access ports in children treated for hematopoietic malignancies. Pediatr Hematol Oncol, 2008. 25(8): p. 751-5.

89. Castagnola E, Fratino G, Valera M, et al., Correlation between "malfunctioning events" and catheter-related infections in pediatric cancer patients bearing tunneled indwelling central venous catheter: results of a prospective observational study. Support Care Cancer, 2005. 13(9): p. 757-9.

90. Cil B E, Radiological placement of chest ports in pediatric oncology patients. Eur Radiol, 2004. 14(11): p. 2015-9.

91. Fratino G, Avanzini S, Molinari A C, et al., Incidence of indwelling central venous catheterrelated complications using the Sri Paran technique for device fixation in children with cancer. Pediatr Surg Int, 2009. 25(7): p. 591-4.

92. Garcia-Teresa M A, Casado-Flores J, Delgado Dominguez M A, et al., Infectious complications of percutaneous central venous catheterization in pediatric patients: a Spanish multicenter study. Intensive Care Med, 2007. 33(3): p. 466-76.

93. Handrup M M, Moller J K, Frydenberg M, et al., Placing of tunneled central venous catheters prior to induction chemotherapy in children with acute lymphoblastic leukemia. Pediatr Blood Cancer, 2010. 55(2): p. 309-13.

94. Hengartner H, Berger C, Nadal D, et al., Port-A-Cath infections in children with cancer. Eur J Cancer, 2004. 40(16): p. 2452-8.

95. Krishnaiah A, Soothill J, Wade A, et al., Central venous catheter-associated bloodstream infections in a pediatric intensive care unit: effect of the location of catheter insertion. Pediatr Crit Care Med, 2012. 13(3): p. e176-80.

96. Ruggiero A, Barone G, Margani G, et al., Groshong catheter-related complications in children with cancer. Pediatr Blood Cancer, 2010. 54(7): p. 947-51.

97. Haumont D, de Beauregard V G, Van Herreweghe I, et al., A new technique for transumbilical insertion of central venous silicone catheters in newborn infants. Acta Paediatr, 2008. 97(7): p. 988-90. 
98. Arnts I J, Bullens L M, Groenewoud J M, et al., Comparison of complication rates between umbilical and peripherally inserted central venous catheters in newborns. J Obstet Gynecol Neonatal Nurs, 2014. 43(2): p. 205-15.

99. Unal S, Ekici F, Cetin, II, et al., Heparin infusion to prevent umbilical venous catheter related thrombosis in neonates. Thromb Res, 2012. 130(5): p. 725-8.

100. Adeb M, Baskin K M, Keller M S, et al., Radiologically placed tunneled hemodialysis catheters: a single pediatric institutional experience of 120 patients. J Vasc Interv Radiol, 2012. 23(5): p. 604-12.

101. Advani S, Reich N G, Sengupta A, et al., Central line-associated bloodstream infection in hospitalized children with peripherally inserted central venous catheters: extending risk analyses outside the intensive care unit. Clin Infect Dis, 2011. 52(9): p. 1108-1115.

102. Brooker R W and Keenan W J, Catheter related bloodstream infection following PICC removal in preterm infants. J Perinatol, 2007. 27(3): p. 171-4.

103. Gaballah M, Krishnamurthy G, Keller M S, et al., Single-incision technique for placement of tunneled internal jugular vein vascular access in children. Pediatr Radiol, 2014.

104. Hoang V, Sills J, Chandler M, et al., Percutaneously inserted central catheter for total parenteral nutrition in neonates: complications rates related to upper versus lower extremity insertion. Pediatrics, 2008. 121(5): p. e1152-9.

105. Jumani K, Advani S, Reich N G, et al., Risk factors for peripherally inserted central venous catheter complications in children. JAMA Pediatr, 2013. 167(5): p. 429-35.

106. Milstone A M, Reich N G, Advani S, et al., Catheter dwell time and CLABSIs in neonates with PICCs: a multicenter cohort study. Pediatrics, 2013. 132(6): p. e1609-15.

107. Piper H G, de Silva N T, Amaral J G, et al., Peripherally inserted central catheters for longterm parenteral nutrition in infants with intestinal failure. J Pediatr Gastroenterol Nutr, 2013. 56(5): p. 578-81.

108. Raj A, Bertolone S, Bond S, et al., Cathlink 20: a subcutaneous implanted central venous access device used in children with sickle cell disease on long-term erythrocytapheresis--a report of low complication rates. Pediatr Blood Cancer, 2005. 44(7): p. 669-72.

109. Reyes J, Habash M, and Taylor R, Femoral venous catheters are not associated with higher rates of infection in the pediatric critical care population. American Journal of Infection Control, 2012. 40: p. 43-7.

110. Sengupta A, Lehmann C, Diener-West M, et al., Catheter duration and risk of CLA-BSI in neonates with PICCs. Pediatrics, 2010. 125(4): p. 648-53.

111. Titapiwatanakun R, Moir C, Pruthi R K, et al., Central venous access devices for paediatric patients with haemophilia: a single-institution experience. Haemophilia, 2009. 15(1): p. 168-74.

112. Toh L M, Mavili E, Moineddin R, et al., Are cuffed peripherally inserted central catheters superior to uncuffed peripherally inserted central catheters? A retrospective review in a tertiary pediatric center. $J$ Vasc Interv Radiol, 2013. 24(9): p. 1316-22.

113. Van Winkle P, Whiffen T, and Liu I L, Experience using peripherally inserted central venous catheters for outpatient parenteral antibiotic therapy in children at a community hospital. Pediatr Infect Dis J, 2008. 27(12): p. 1069-72.

114. Wong J, Dow K, Shah P S, et al., Percutaneously placed central venous catheter-related sepsis in Canadian neonatal intensive care units. Am J Perinatol, 2012. 29(8): p. 629-34.

115. Abedin S and Kapoor G, Peripherally inserted central venous catheters are a good option for prolonged venous access in children with cancer. Pediatr Blood Cancer, 2008. 51(2): p. 251-5.

116. Eisenstein I, Tarabeih M, Magen D, et al., Low infection rates and prolonged survival times of hemodialysis catheters in infants and children. Clin J Am Soc Nephrol, 2011. 6(4): p. 7938. 
117. Hsu J F, Tsai M H, Huang H R, et al., Risk factors of catheter-related bloodstream infection with percutaneously inserted central venous catheters in very low birth weight infants: a center's experience in Taiwan. Pediatr Neonatol, 2010. 51(6): p. 336-42.

118. Hatakeyama N, Hori T, Yamamoto M, et al., An evaluation of peripherally inserted central venous catheters for children with cancer requiring long-term venous access. Int J Hematol, 2011. 94(4): p. 372-7.

119. Hung M C, Chen C J, Wu K G, et al., Subcutaneously implanted central venous access device infection in pediatric patients with cancer. J Microbiol Immunol Infect, 2009. 42(2): p. 166-71.

120. Levy I, Bendet M, Samra Z, et al., Infectious complications of peripherally inserted central venous catheters in children. Pediatr Infect Dis J, 2010. 29(5): p. 426-9.

121. Matsuzaki A, Suminoe A, Koga Y, et al., Long-term use of peripherally inserted central venous catheters for cancer chemotherapy in children. Support Care Cancer, 2006. 14(2): p. 153-60.

122. Shen G, Gao Y, Wang Y, et al., Survey of the long-term use of peripherally inserted central venous catheters in children with cancer: experience in a developing country. $J$ Pediatr Hematol Oncol, 2009. 31(7): p. 489-92.

123. Tsai M H, Lien R, Wang J W, et al., Complication rates with central venous catheters inserted at femoral and non-femoral sites in very low birth weight infants. Pediatr Infect Dis $J$, 2009. 28(11): p. 966-70.

124. Tsai M H, Chu S M, Lien R, et al., Complications associated with 2 different types of percutaneously inserted central venous catheters in very low birth weight infants. Infect Control Hosp Epidemiol, 2011. 32(3): p. 258-66.

125. Yacobovich J, Ben-Ami T, Abdalla T, et al., Patient and central venous catheter related risk factors for blood stream infections in children receiving chemotherapy. Pediatric Blood \& Cancer, 2014: p. n/a-n/a.

126. Chopra V, Anand S, Hickner A, et al., Risk of venous thromboembolism associated with peripherally inserted central catheters: a systematic review and meta-analysis. Lancet, 2013. 382(9889): p. 311-25.

127. Chopra V, O'Horo J C, Rogers M A, et al., The risk of bloodstream infection associated with peripherally inserted central catheters compared with central venous catheters in adults: a systematic review and meta-analysis. Infect Control Hosp Epidemiol, 2013. 34(9): p. 90818.

128. Sagana R and Hyzy R C, Achieving zero central line-associated bloodstream infection rates in your intensive care unit. Crit Care Clin, 2013. 29(1): p. 1-9.

129. Worth L J and McLaws M L, Is it possible to achieve a target of zero central line associated bloodstream infections? Curr Opin Infect Dis, 2012. 25(6): p. 650-7.

130. Ardura M I, Lewis J, Tansmore J L, et al., Central catheter-associated bloodstream infection reduction with ethanol lock prophylaxis in pediatric intestinal failure: broadening quality improvement initiatives from hospital to home. JAMA Pediatr, 2015. 169(4): p. 324-31.

131. Kleidon T, Illing A, Fogarty G, et al., Improving the central venous access devices maintenance process to reduce associated infections in paediatrics: evaluation of a practical, multi-faceted quality-improvement initiative. Healthcare Infection, 2015. 20(2): p. 46-53.

132. Bundy D G, Gaur A H, Billett A L, et al., Preventing CLABSIs among pediatric hematology/oncology inpatients: national collaborative results. Pediatrics, 2014. 134(6): p. e1678-85.

133. McGee D C and Gould M K, Preventing complications of central venous catheterization. $N$ Engl J Med, 2003. 348(12): p. 1123-33.

134. Dudeck M A, Edwards J R, Allen-Bridson K, et al., National Healthcare Safety Network report, data summary for 2013, Device-associated Module. Am J Infect Control, 2015. 43(3): p. 206-221. 
Figure 1: PRISMA flow chart of study selection

\section{Supplementary Figures}

Figure 2: Forest plot showing the pooled, weighted proportion of CVAD failure across CVAD type Random effects meta-analysis showing the individual and pooled weighted proportion of CVAD failure; stratified by CVAD type

Figure 3: Forest plot showing the pooled, weighted incidence rate of CVAD failure per 1,000 catheter days across CVAD type Inverse variance meta-analysis showing the individual and pooled incidence rate of CVAD failure per 1,000 catheter days; stratified by CVAD type

Figure 4: Forest plot showing the pooled, weighted proportion of CVAD-associated bloodstream infection across CVAD type

Random effects meta-analysis showing the individual and pooled weighted proportion of CVADassociated bloodstream infection; stratified by CVAD type

Figure 5: Forest plot showing the pooled, weighted incidence rate of CVAD-associated bloodstream infection per 1,000 catheter days across CVAD type

Inverse variance meta-analysis showing the individual and pooled incidence rate of CVADassociated bloodstream infection per 1,000 catheter days; stratified by CVAD type 